\title{
Protein kinase $C$ and rho activated coiled coil protein kinase 2 (ROCK2) modulate Alzheimer's APP metabolism and phosphorylation of the Vps10-domain protein, SorL1
}

\author{
Rachel F Lane 1* $^{*}$ Joshua W Gatson², Scott A Small ${ }^{3}$, Michelle E Ehrlich ${ }^{1,4,5}$, Sam Gandy ${ }^{1,6,7}$
}

\begin{abstract}
Background: Generation of the amyloid $\beta$ (AB) peptide of Alzheimer's disease (AD) is differentially regulated through the intracellular trafficking of the amyloid $\beta$ precursor protein (APP) within the secretory and endocytic pathways. Protein kinase C (PKC) and rho-activated coiled-coil kinases (ROCKs) are two "third messenger" signaling molecules that control the relative utilization of these two pathways. Several members of the Vps family of receptors (Vps35, SorL1, SorCS1) play important roles in post-trans-Golgi network (TGN) sorting and generation of APP derivatives, including $A \beta$ at the TGN, endosome and the plasma membrane. We now report that Vps10domain proteins are candidate substrates for PKC and/or ROCK2 and act as phospho-state-sensitive physiological effectors for post-TGN sorting of APP and its derivatives.

Results: Analysis of the SorL1 cytoplasmic tail revealed multiple consensus sites for phosphorylation by protein kinases. SorL1 was subsequently identified as a phosphoprotein, based on sensitivity of its electrophoretic migration pattern to calf intestine alkaline phosphatase and on its reaction with anti-phospho-serine antibodies. Activation of PKC resulted in increased shedding of the ectodomains of both APP and SorL1, and this was paralleled by an apparent increase in the level of the phosphorylated form of SorL1. ROCK2, the neuronal isoform of another protein kinase, was found to form complexes with SorL1, and both ROCK2 inhibition and ROCK2 knockdown enhanced generation of both soluble APP and A .
\end{abstract}

Conclusion: These results highlight the potential importance of SorL1 in elucidating phospho-state sensitive mechanisms in the regulation of metabolism of APP and A $\beta$ by PKC and ROCK2.

\section{Introduction}

Aberrant processing of the Alzheimer's amyloid precursor protein (APP) is believed to underlie some forms of Alzheimer's disease (AD), leading to increased generation and/or decreased clearance of amyloid beta 42 (A 342$)$. APP is differentially processed within discrete intracellular compartments. Metabolism of APP by either the endocytic pathway or the constitutive secretory pathway is regulated on a moment to moment basis by the integration of intercellular and intracellular signals, including membrane depolarization and first

\footnotetext{
* Correspondence: rachel.lane@mssm.edu

'Department of Neurology, Mount Sinai School of Medicine, New York NY 10029, USA

Full list of author information is available at the end of the article
}

messenger activation of their cognate receptors [for reviews, see 1-3]. Second messengers such as calcium and cyclic AMP act via third messengers that are enzymes that control protein phosphorylation (i.e., protein kinases and protein phosphatases). Third messengers enzymes implicated in regulating APP metabolism include protein kinase $C$ (PKC; [4-9]), protein phosphatases 1 and 2A (PP1, PP2A; [4-7]), extracellular signal regulated protein kinase (ERK; [10]), casein kinases (CK; [11]), Janus kinase (JNK; $[12,13])$, and rho-associated coiled-coil protein kinases (ROCK; [14]).

We have a longstanding interest in identifying the important phospho-state-sensitive physiological effectors that are targets for these third messengers $[4,8,15]$. The obvious candidates for identities of phospho-state sensitive 
molecules relevant to APP metabolism include: (i) APP itself $[4,15]$; (ii) the various APP sorting and trafficking proteins ([8]; this manuscript); and (iii) the secretases $[16,17]$. APP phosphorylation at serine 655 was discovered in 1988 [4], and its physiological role includes regulation of the interaction of APP with the retromer trafficking complex [15] and activation of PKC is associated with increased retromer-mediated transport of APP to the $T G N$ and decreased $A \beta$ generation [15]. In addition to phosphorylation of APP at serine 655, additional phosphoacceptor sites have been discovered. Suzuki and colleagues have documented an important role of the phosphorylation state of APP threonine 668 in APP maturation and sorting [reviewed in [3]] while phosphorylation of the cytoplasmic tail at tyrosine 682 and/or 687 has been reported to regulate release of the APP intracellular domain (AICD; [18,19].

APP retrograde trafficking to the $T G N$ and regulation of $A \beta$ generation is dependent on SorL1 interaction with the core component of the retromer complex, Vps35 [20,21]. In addditon, we have recently implicated a second member of the Vps10 family, SorCS1 in retromer-mediated regulation of $A \beta$ generation [22]. In the current study, we have begun investigating the possibilities that some of these Vps10-domain proteins are also important phospho-state-sensitive modulators of postTGN APP metabolism. Herein, we report: (i) that SorL1 is a phosphoprotein; (ii) that both PKC and ROCK2 interact with SorL1; and (iii) that modulation of ROCK2 activity regulates generation of $A \beta$.

\section{Results and Discussion}

\section{The subcellular trafficking itinerary for APP}

In order to understand how PKC, ROCK, and SorL1 might play important roles in APP metabolism, one must understand the details of APP sorting and processing in post TGN compartments. Following exit of mature APP from the TGN, some APP molecules are conveyed by the secretory pathway to the plasma membrane where APP can encounter and be cleaved within the $A \beta$ domain by one of the $\alpha$-secretases; this is the nonamyloidogenic (non-A $\beta$-forming) pathway) (for review see, [2]). $\alpha$-secretase cleavage of APP results in the formation of a cell retained carboxyl terminal fragment $(\alpha-C T F)$ and in the shedding of the APP ectodomain, which is also known as soluble APP $\alpha$ (sAP $\alpha)$. APP molecules that do not enter the secretory pathway are targeted to a TGN-localized population of clathrincoated vesicles (CCVs). From here, APP is conveyed into the endocytic pathway where APP can encounter and be cleaved by the $\beta$-secretase, BACE (for $\beta$-APP site cleaving enzyme). BACE cleavage of APP occurs primarily in endosomes, and this step defines APP entry into the potentially amyloidogenic ( $\mathrm{A} \beta$-forming) pathway, resulting in formation of a soluble sAPP $\beta$ fragment and a membrane bound $\beta \mathrm{CTF}$, amino terminus of which is identical to that of $A \beta$ [for review, see [2]]. APP not cleaved at the plasma membrane by the $\alpha$-secretases can be reinternalized into the endocytic pathway thereby also entering the amyloidogenic pathway.

Within the endosomal system, the $\alpha$ - and $\beta$-CTFs serve as the proximate substrates for $\gamma$-secretase. $\gamma$-secretase cleavage of the $\alpha$-CTF gives rise to secreted nonamyloidogenic p3 and an intracytoplasmic fragment, AICD (APP intracellular domain), whereas $\gamma$-secretase cleavage of the $\beta$-CTF gives rise to $A \beta$ and also to AICD. The $\gamma$-secretase cleavage is especially important since this is the step where the major speciation occurs, giving rise to the heterogeneous carboxyl termini that define $A \beta 40$ and $A \beta 42$. Oligomerization and accumulation of $A \beta 42$ are believed to be the key inciting events in causation of $\mathrm{AD}$. Ultimately reducing the time of residence of APP and/or the $\beta$-CTF within the endocytic pathway decreases $A \beta$ generation. Retromer-mediated trafficking of APP and/or its CTFs away from the endosome to the TGN plays a key role in modulating residence time for APP and/or its CTFs in the endocytic system [for review, see [2]]. As shown by Small et al [20], the retromer is especially important in sporadic AD because hippocampal subregion-specific progression of $\mathrm{AD}$ pathology is linked to a deficiency in the core component of the retromer, Vps35 [20].

Post-TGN sorting and metabolism of APP is controlled by at least two Vps10 domain proteins, SorL1 [2,23-27] and SorCS1 [22]. At least one action of SorL1 involves its function as a retromer adaptor or receptor, linking APP to Vps35 [2,21]. When the SorL1-APP interaction is abolished by mutation of the FANSHY domain in the cytoplasmic tail of SorL1 that specifies its binding to Vps35, that FANSHY domain mutant is unable to regulate APP metabolism [21]. In addition to its role in retromer-mediated retrieval of APP, another model suggests that SorL1 plays a gating or retention function for APP at the TGN [27]. The molecular mechanism for this interaction at the TGN has been suggested to involve SorL1 and APP interacting together with the adaptors GGA and PACS- 1 to control exit of APP into the amyloidogenic and non-amyloidogenic pathways [27]. The two models for SorL1 action (the retromer model and the TGN retention model) are not mutually exclusive, and their relative importance remains unknown.

\section{Regulation of APP sorting and metabolism by protein phosphorylation}

The first and second messengers linked to APP processing lead to activation of third messenger protein kinases and protein phosphatases. PKC and ROCK have 
emerged as especially interesting since they have different effects on APP metabolism, perhaps due to differential effects on sorting of APP and/or its derivatives. PKC activates the $\alpha$-secretase pathway [4-8], while ROCK inhibits the $\alpha$-secretase pathway [14]. These protein kinases and protein phosphatases converge on physiological effector substrate phosphoproteins, and the phosphorylation status of those effectors typically specifies their states of activation. Identification of the phosphostate sensitive effector molecules that modulate APP metabolism has been challenging. Vps10-domain proteins are outstanding candidates for such effectors. Since SorL1 has been proposed [2] and recently demonstrated [21] to act as an adaptor that links APP to the retromer complex, we began our study of phospho-state regulated APP metabolism by Vps10 domain proteins with the current study of SorL1.

\section{SorL1 is a phosphoprotein}

Phospho-site consensus motif software that identifies the presence of known consensus kinase recognition motifs [28] revealed multiple putative phosphorylation sites in the SorL1 cytoplasmic tail (Figure 1). As mentioned above, in addition to these phospho-acceptor consensus motifs, a motif in the SorL1 cytoplasmic tail has been recently identified that is essential for its interaction with Vps35 [21]. This motif, 2172 Phe-Ala-AsnSer-His-Tyr 2173 (FANSHY), is flanked by consensus sites for PKC phosphorylation at serines 2167 and 2178. The FANSHY domain is of particular relevance since the mutations of this motif that abolish SorL1-Vps35 interaction also abolish the ability of SorL1 to control APP processing [21]. These observations raised the possibility that the phosphorylation status surrounding the FANSHY domain might play a key role in the modulation of APP metabolism by SorL1. In order to begin to test this hypothesis, we first sought to determine whether SorL1 is itself a phosphoprotein.

To determine whether SorL1 is a phosphoprotein, we investigated the electrophoretic migration pattern of SorL1 on low percentage SDS PAGE before and after dephosphorylation. SorL1 immunopositive bands following a mock reaction without calf intestine alkaline phosphatase (CIP) revealed three SorL1 immunopositive species migrating at approximately $250-270 \mathrm{kDa}$ that were independently detected with two different antiSorL1 antibodies. Upon dephosphorylation with CIP, the electrophoretic migration pattern shifted dramatically, and the highest molecular weight species of SorL1 shifted and co-migrated with the lower molecular weight, immature species (Figure 2A). This behavior was consistent with the possibility that SorL1 is a phosphoprotein, and that the slower migrating SorL1 species was modified by the addition of one or more phosphate moieties.

In order to seek independent confirmatory evidence that SorL1 is a phosphoprotein, we employed the ProQ Diamond phospho-stain (Invitrogen) that specifically detects phosphorylated proteins in polyacrylamide gels. ProQ detected multiple bands of approximately $250 \mathrm{kDa}$ that co-migrated with the high molecular weight candidate phospho-SorL1, and these were abolished following treatment with CIP (Figure 2A).

We also sought to determine whether the high molecular weight candidate phospho-SorL1 could be detected with a specific phospho-serine antibody. Immunoblotting of untreated and CIP-treated lysates revealed immunopositive bands with the same migratory pattern

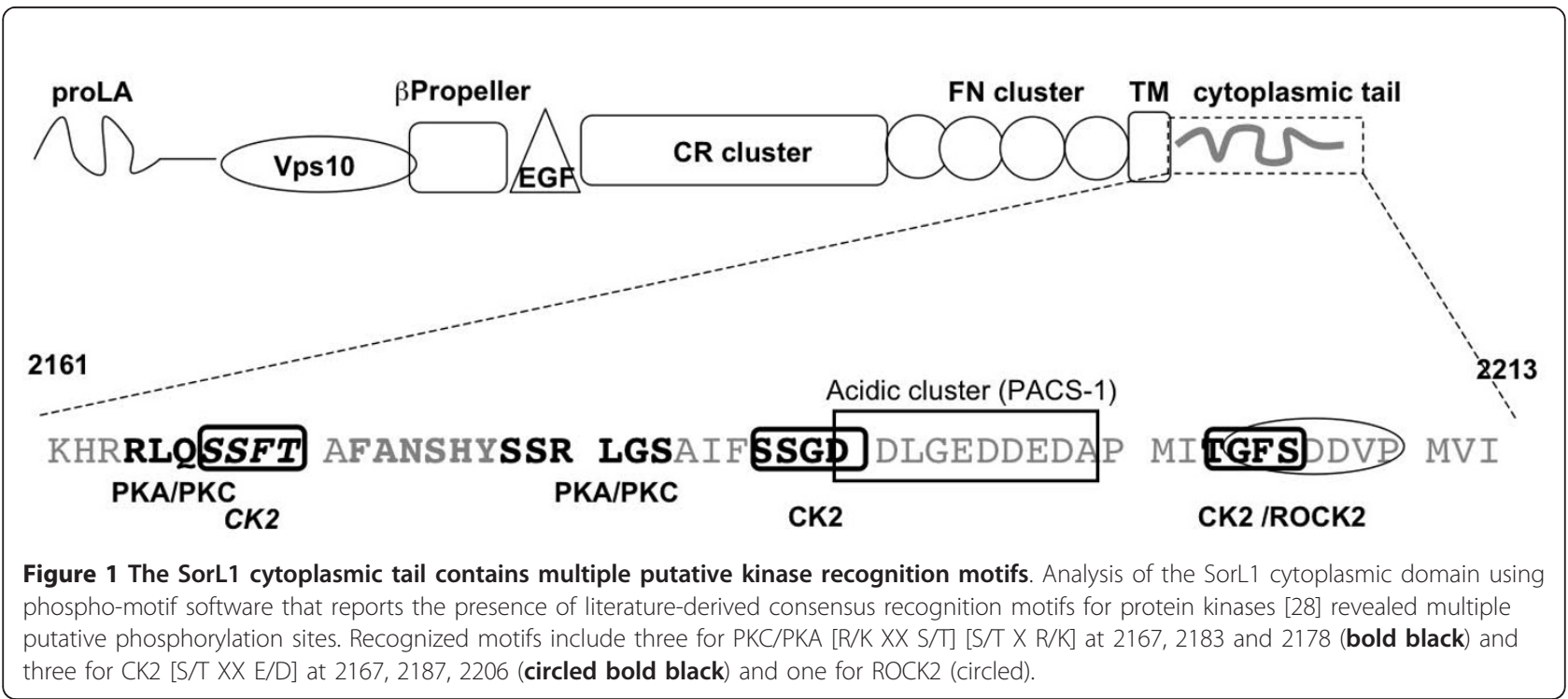




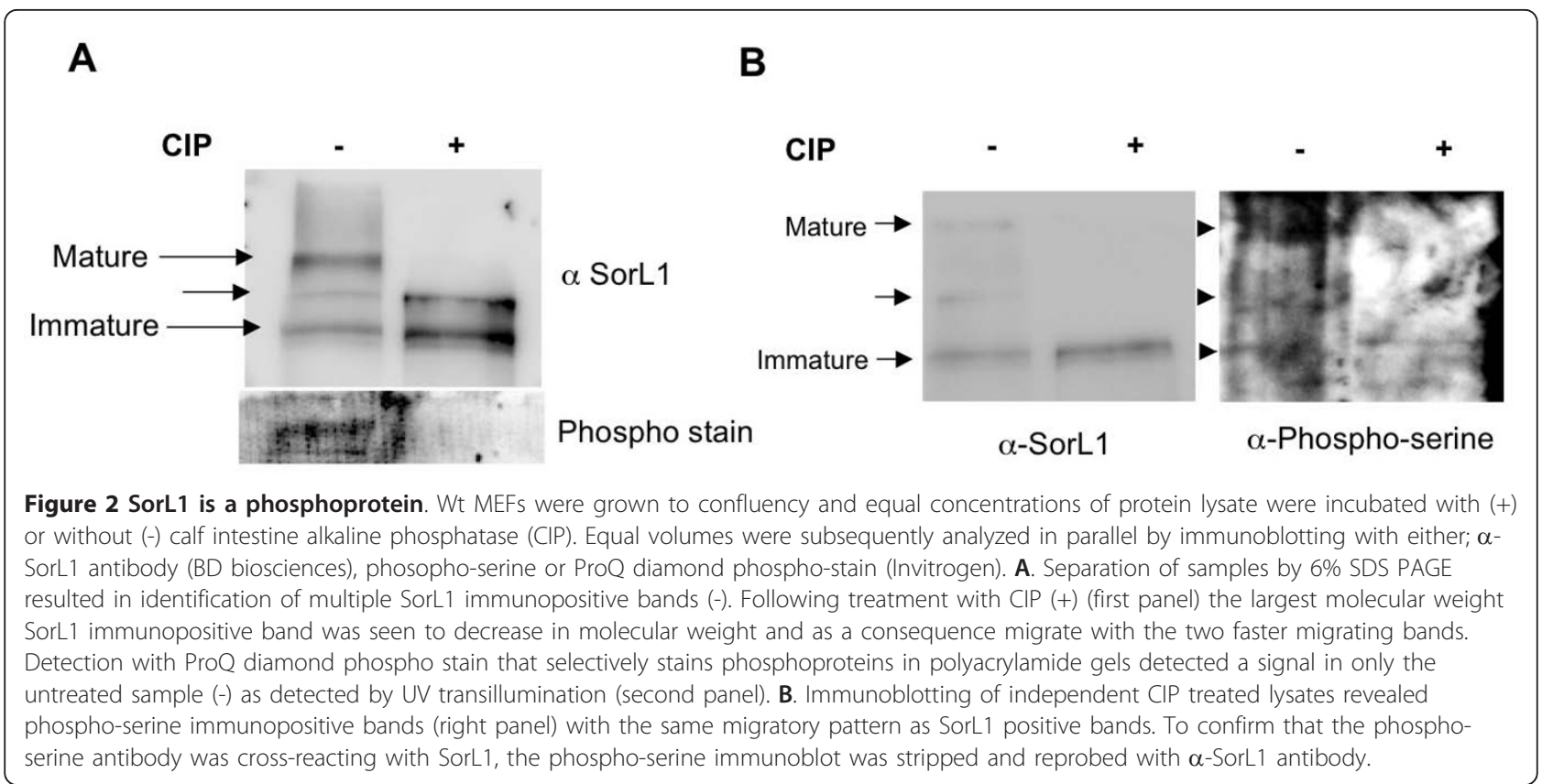

as SorL1. As before, the higher molecular weight SorL1 species were not detected with this antibody following dephosphorylation with CIP (Figure 2B). Taken together, the altered migration of SorL1 following dephosphorylation and the staining with both a phospho-stain and with anti-phospho-serine antibodies support the conclusion that SorL1 is a phosphoprotein.

\section{PKC activation stimulates SorL1 phosphorylation}

PKC activates $\alpha$-secretase-mediated ectodomain shedding of APP, and a number of $\gamma$-secretase substrates, including the Vps10 family members sortilin, SorL1, SorCS1, 2 and $3[8,23]$. Therefore, we sought to determine whether SorL1 phosphorylation was enhanced following stimulation of PKC. As previously reported, phorbol-12,14dibutyrate (PDBu, Sigma), a PKC activator, stimulates ectodomain shedding of APP and other $\gamma$-secretase substrates including SorL1 [8,23] (Figure 3A). Treatment of mouse embryonic fibroblasts (MEFs) with PDBu increased shedding of the ectodomains of SorL1 and APP, and that effect was greater following treatment with the combination of PDBu and okadaic acid (OA), an inhibitor of protein phosphatases 1 and 2A (Figure 3A, B). Interestingly, upon treatment with $\mathrm{PDBu}$, the highest molecular weight SorL1 species (upper two bands) showed increased signal intensity (Figure 3B) and migration of the SorL1 CTF was slightly retarded compared to untreated control (Figure 3A), consistent with the shifts expected for proteins following addition of phosphate moieties.

Taken together with our previous observations, these data suggest that PKC activation results in increased phosphorylation and ectodomain shedding of SorL1. We cannot yet determine how SorL1 shedding influences its ability to regulate $A \beta$. Further study is required to determine whether PKC does indeed directly phosphorylate SorL1, and, if so, whether this addition of phosphate to SorL1 is essential for regulation of soluble SorL1 and APP shedding and/or A $\beta$ generation. The role of the proximity of the FANSHY domain to the potential phosphorylation sites also remains to be explored.

\section{SorL1 interacts with ROCK2}

We have previously reported that ROCK1 inhibits basal and statin-activated $\alpha$-secretase cleavage and shedding of sAPP $\alpha$ [14]. While ROCK1 is highly expressed in lung, heart, stomach, liver, kidney, placenta, and testis, it is the ROCK2 isoform that is more abundant in the heart and brain [29], suggesting that the actions of ROCK in the brain may be mediated primarily by the ROCK2 isoform. We investigated the possibility that ROCK2 interacts with SorL1. Immunoprecipitation of SorL1 resulted in co-immunoprecipitation of ROCK2 (Figure 4A). To confirm this interaction, we performed the reverse immunoprecipitation and, indeed, SorL1 was recovered from the anti-ROCK2 immunoprecipitate but not from the control IgG immunoprecipitate (Figure 4B). Though we did not assess whether ROCK2 could regulate phosphorylation of SorL1, Herskowitz et al (2010) [30] recently reported that ROCK2 modulates SorL1 phosphorylation, consistent with our observation that the two molecules can exist as a complex. As with PKC phosphorylation of the SorL1 cytoplasmic tail, the possible phospho-state-sensitive modulation 


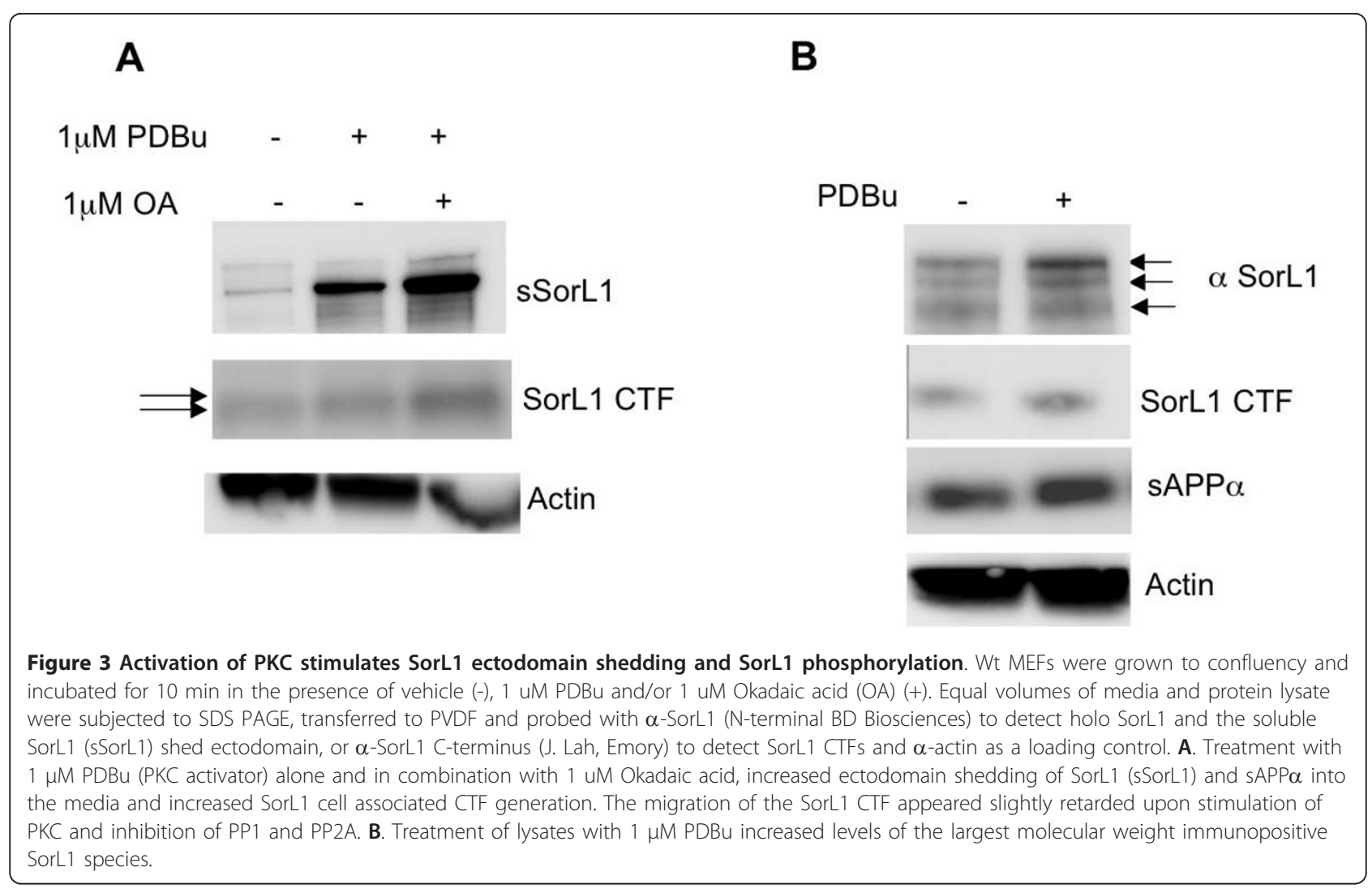

of FANSHY-mediated SorL1-Vps35 interaction remains to be elucidated. Given their differential effects on APP metabolism [[14], this paper], it is tempting to speculate that differential phosphorylation of SorL1 by PKC and/or ROCK1 may be involved.

\section{Inhibition of ROCK 2 increases $A \beta$ generation}

Having identified ROCK2 as a SorL1 binding protein, we next sought to determine the effect of ROCK2 activity on $\mathrm{A} \beta$ generation. Treatment of mouse N2a APPSwe $\triangle$ 9PS1 neuroblastoma cells for 100 mins with the

\section{A}
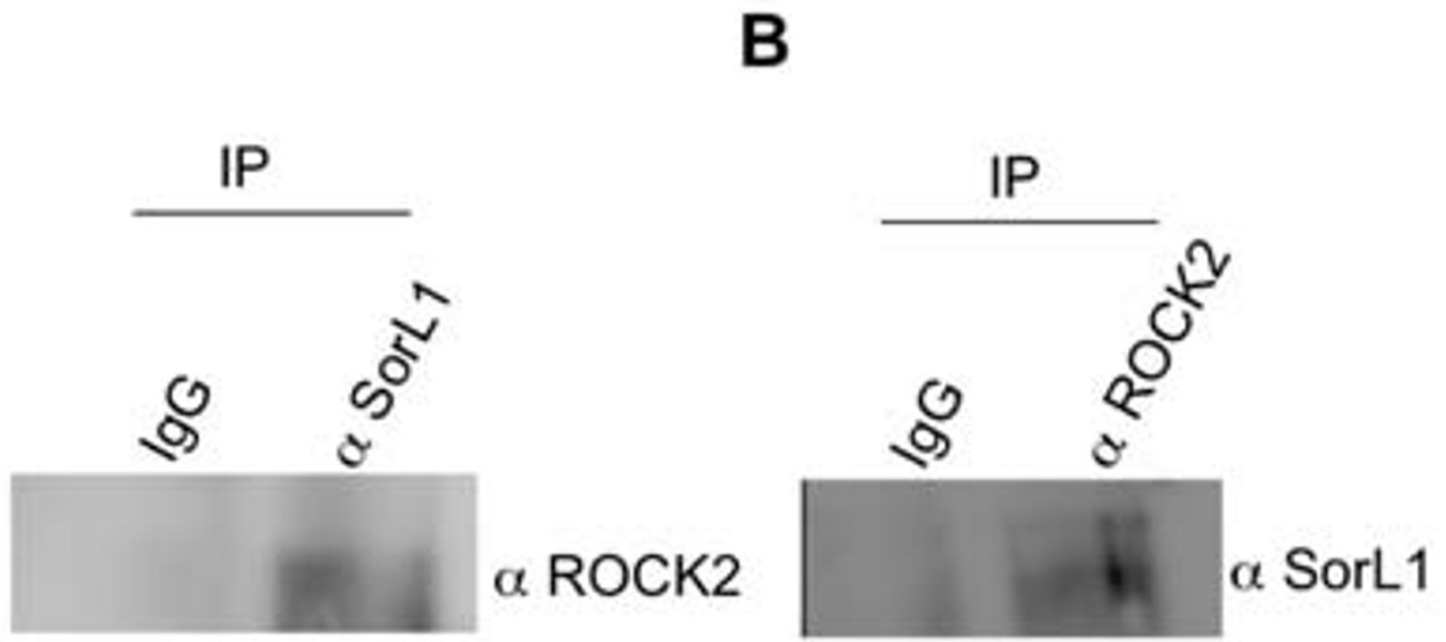

Figure 4 SorL1 interacts with ROCK2. A. Endogenous SorL1 was immunoprecipitated from Wt MEFs using $\alpha$ SorL1 antibody (BD biosciences) or control IgG. Immunooprecipitates were subjected to SDS-PAGE, transferred to PVDF membranes and probed $\alpha$-ROCK2. ROCK2 was detected in the $\alpha$-SorL1 precipitate. B. Immunoprecipitation of ROCK2 using $\alpha$-ROCK2 antibody (BD biosciences) resulted in co-immunoprecipitation of SorL1. 
selective ROCK2 inhibitor RL1 $(100 \mathrm{nM})$ resulted in increased generation of both $\mathrm{A} \beta 40(p=0.007)$ and A $\beta 42(p=0.03)$ (Figure 5A). To confirm this observation and rule out off-target affects of RL1, we next assessed the effect of specific shRNA lentiviral mediated knockdown of ROCK2. Transduction with shRNA ROCK2 (but not with the scrambled shRNA control) resulted in increased generation of both $\mathrm{A} \beta$ and sAPP $\alpha$ in 4 different shRNA ROCK2 reactions (Figure 5B, C).

Like most protein kinases, ROCK autophosphorylates itself, and the phospho-form is the more active form. The absence of perfect dose-dependence between ROCK 2 knockdown and $A \beta$ generation in Figure 5 may be due to differences in ROCK2 specific activity related to differences in phosphorylation status. PhosphoROCK2-specific antibodies are being developed to clarify this point.

While the inhibitor and shRNA sequence used in this study are specific for ROCK2 when known substrates are studied, the two isoforms show significant homology, and we therefore cannot completely rule out non-specific effects and/or effects mediated by ROCK1.

\section{Conclusions}

The involvement of the Rho/ROCK pathway in AD has been reported by several research groups $[14,31,32]$. However, the mechanisms by which ROCK regulates APP processing and $A \beta$ generation are not well understood.

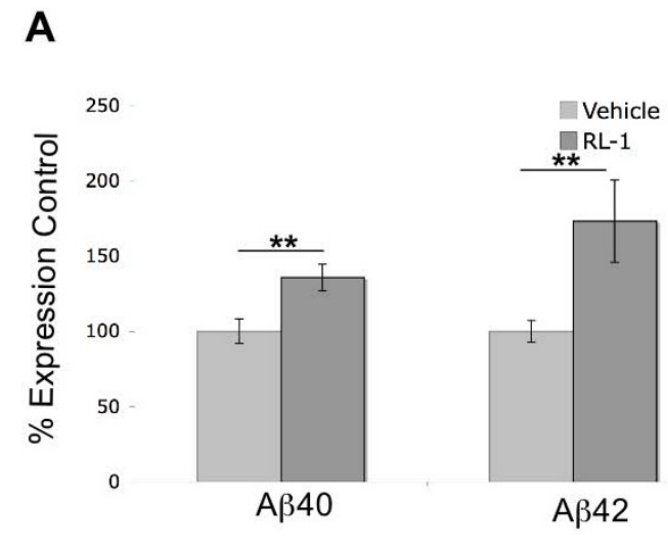

B
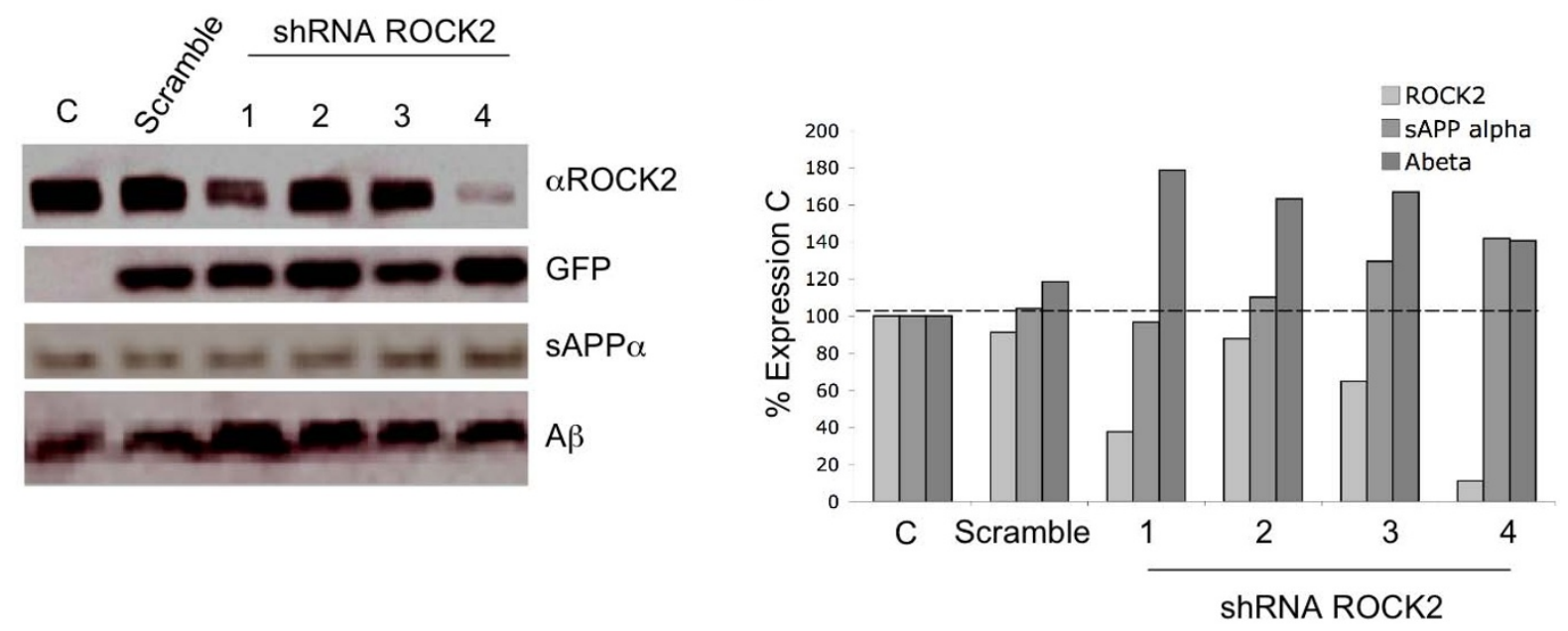

Figure $\mathbf{5}$ Inhibition of ROCK2 increases $\mathbf{A} \boldsymbol{\beta} 40$ and $\mathbf{A} \mathbf{\beta} \mathbf{4 2}$ generation. $\mathbf{A}$. N2A APPSwe $\triangle 9 P S 1$ cells were grown to confluency and treated with vehicle or $100 \mathrm{nM} \mathrm{RL-1}$ for 60 mins. After 60 mins equal volumes of media were collected and $A \beta 40$ and $A \beta 42$ levels were determined by sandwich ELISA (Wako). A $\beta 40(p=0.007)$ and A $342(p=0.03)$ levels were increased following treatment with $100 \mathrm{nM}$ of the specific ROCK2 inhibitor, RL-1. Data was collected in duplicate or triplicate from 3 independent iterations $\left({ }^{*} p>0.05{ }^{* *} p<0.01\right)$. B. N2A APP Swe $\triangle 9 P S 1$ cells were transduced with non-specific scrambled shRNA or 4 different shRNA ROCK2. 72 hrs post transduction media and cell lysates were collected and snap frozen. Equal volume and concentrations of media and lysate respectively were analyzed by SDS PAGE and transferred to PVDF. Lysates were analyzed for ROCK2 and GFP as a control for transduction efficiency and media analyzed for sAPP $\alpha$ and A $\beta$. Protein levels are expressed as percentage of non-transduced C (C). Knockdown of ROCK2 resulted in increased generation of A $\beta$ and sAPP $\alpha$ with all shRNA ROCK2 viruses analyzed but not after transduction with the scrambled non-specific control shRNA. 
While we previously demonstrated that expression of a dominant negative (DN) ROCK1 increased $\mathrm{SAPP} \alpha, \mathrm{A} \beta$ generation was not addressed in that system [14]. In the current study, we observed that shRNA mediated knockdown of ROCK2 resulted in increased release of both sAPP $\alpha$ and A $\beta$. While the increase in sAPP $\alpha$ is consistent with our earlier ROCK1 DN data [14] the increase in $A \beta$ is unexpected. One explanation may lie in the mechanism and subcellular localization by which SorL1 regulates $A \beta$ generation. This is the first example of a protein kinase that regulates both $\alpha$ - and $\beta$-secretase pathways in parallel. These data can be construed to fit either the TGN model of SorL1 action or the retromer model. SorL1phospho-state-dependent dissociation of APP and/or CTFs bound in complexes localized to either the TGN or to the retromer would be expected to increase substrate available for generation of $\mathrm{APP} \alpha$ and $\mathrm{A} \beta$. However, the net effect on APP metabolism would also have to take into account any actions related to the phosphorylation status of APP serine-655 [15]. Additional data will be required in order to develop a model that takes into account all relevant molecules and their phosphorylation status.

Further investigation is required to establish the roles of ROCK2, PKC and various Vps10 domain proteins in the phospho-state sensitive generation of $A \beta$ generation. Work by Small et al (2005) [20], Muhammad et al., (2009) [33], and Andersen et al., (2010) [21] have demonstrated the importance of the retromer, and it will be important to elucidate the possible role of direct phosphorylation of Vps35 and Vps10-domain receptors. Ultimately the regulation of APP metabolism by protein phosphorylation provides an indirect pathway for therapeutic modulation of $A \beta$ generation. The recent evidence that $A \beta$ plays a physiological role in normal memory formation and the recent failure of a $\gamma$-secretase inhibitor in phase 3 clinical trials has pointed up the necessity for fine regulation of $A \beta$ metabolism in order to balance the requirement for $A \beta$ levels that support memory function against the therapeutic goal of avoiding $\mathrm{A} \beta$ accumulation. Targeting a protein phosphorylation regulatory pathway for APP metabolism may enable finer adjustment than that achievable by direct inhibition of $\beta$ - or $\gamma$-secretase.

Balancing between $\alpha$ and $\beta$ secretase pathways physiology and pathophysiology will be especially important given the likelihood that $A \beta$-lowering drugs may well be administered prophylactically to people with intact cognition and then maintained for several decades.

\section{Materials and methods Antibodies}

$\alpha$-SorL1 (N-terminal, BD Biosciences), $\alpha$-ROCK2 (BDBiosciences), $\alpha$-actin (Sigma), $\alpha$-phospho-serine antibody (Abcam), $\alpha$-GFP (Sigma), 6E10 (Covance, sAPP $\alpha$ and $\mathrm{A} \beta$ ), anti-mouse, anti-rabbit HRP conjugates (Vector Labs) were purchased. $\alpha$-SorL1 (C-terminal, gift from J. Lah, Emory University) to detect SorL1 Cterminal fragments (CTFs), Ab369 (C-terminal APP antibody) to detect human holoAPP and C-terminal fragments [5] were kindly donated. Pro-Q Diamond phosphoprotein Gel stain (Invitrogen) was used to detect putative phosphorylated proteins as per the manufacturers instructions.

\section{Cell culture studies}

Human embryonic kidney 293 cells and wild type (Wt) mouse embryonic fibroblasts (MEF) cells were cultured at $37^{\circ} \mathrm{C} / 5 \% \mathrm{CO}_{2}$ in growth medium (DMEM, $10 \% \mathrm{FBS}$, $1 \%$ penicillin/streptomycin, 1\%L-glutamine Gibco). Mouse N2a neuroblastoma cells stably co-transfected with the FAD mutant forms of APP and PS1 (N2a APPSwe $\triangle 9 P S 1$ ) (gift from G. Thinakaran, University of Chicago, Chicago, IL.), were maintained in DMEM supplemented with $10 \% \mathrm{FBS}$, penicillin $(100 \mathrm{U} / \mathrm{ml})$, streptomycin $(100 \mathrm{mg} / \mathrm{ml})$, geneticin $(200 \mathrm{mg} / \mathrm{ml})$, and puromycin $(5 \mathrm{mg} / \mathrm{ml})$.

Wildtype MEFs were used for SorL1 phosphorylation and processing studies, N2a APPSwe $\triangle E 9$ PS1 were used for investigation for pharmacological inhibition of ROCK2 and shRNA knockdown studies and HEK 293t cells were used to generate scramble shRNA and ROCK2 shRNA lentivirus.

\section{Pharmacological studies}

Wildtype MEFs were treated in serum free DMEM with either PDBu (PKC activator, Sigma), okadaic acid (OA) (protein phosphatase 1 (PP1) and 2A (PP2A), Sigma) or 4,5,6,7-Tetrabromobenzotriazole (TBB) (CK2 inhibitor Tocris Biosciences). At specified time points media was collected and snap frozen. Cells were harvested in RIPA buffer $(50 \mathrm{mM}$ Tris $\mathrm{HCl} \mathrm{pH}$ 7.5, $100 \mathrm{mM} \mathrm{NaCl}, 1 \mathrm{mM}$ EDTA, $1 \mathrm{mM}$ DTT, 1\% NP40, $0.2 \mathrm{mM}$ PMSF, $0.2 \mathrm{mM}$ $\mathrm{Na}_{3} \mathrm{VO}_{4}, 50 \mathrm{mM} \mathrm{NaF}, 10 \mathrm{mM} \mathrm{Na} \mathrm{P}_{2} \mathrm{O}_{7}$ plus Roche complete EDTA-free protease inhibitor tablet) using 5 cycles of $20 \mathrm{~s}$ vortex $/ 5 \mathrm{~min}$ ice incubation, with the exception of alkaline phosphatase (CIP) studies where protein phosphatases were excluded from the RIPA buffer. Cell debris was removed by centrifugation at $4{ }^{\circ} \mathrm{C}$ at $10,000 \times g$ for $15 \mathrm{~min}$.

Protein concentrations from cell lysates and media were determined using the Bio-Rad Protein Determination Kit. Absorbance was read at $595 \mathrm{~nm}$ using a BioRad Microplate Reader (680XR) and analyzed using Microplate Manager v5.2.1. Samples were subsequently prepared in $5 \times$ Laemmli buffer and boiled at $95^{\circ} \mathrm{C}$ for 5 minutes. For analyses of different SorL1 immunoreactive species, lysates and media were separated by tris-glycine $6 \%$ and $10 \%$ SDS PAGE gels respectively, transferred 
to PVDF and immunoblotted using $\alpha$-SorL1 (BD biosciences) which recognizes an epitope in the SorL1 shed ectodomain to detect 1) holo-SorL1 in lysates and 2) soluble SorL1 (sSorL1) in media samples and $\alpha$-SorL1 (J. Lah, Emory) to detect the SorL1 CTF.

To detect putative phosphorylated immunopositive SorL1 species, $60 \mu \mathrm{g}$ protein lysate was treated with vehicle or CIP (NEB) according to manufacturers instructions and subsequently prepared in $1 \mathrm{X}$ Laemmli buffer and analyzed as above.

N2a APPSwe PS1 were grown to 70\% confluency, treated with $100 \mathrm{nM}$ of the selective ROCK2 inhibitor RL-1 or vehicle control (DMSO) for 60 minutes in serum free DMEM. At 60 mins post treatment, equal volume of media were collected and snap frozen and $A \beta 40$ and $A \beta 42$ levels were determined by the human $A \beta$ (40 and 42) ELISA kit (WAKO), according to the manufacturer's instructions. Absorbance was read at $450 \mathrm{~nm}$ using a BioRad microplate reader. Results were normalized to control ( $\mathrm{pg} / \mathrm{mol})$ and expressed as percentage expression of control.

\section{Immunoprecipitations}

Wildtype MEFs were grown to confluency and lysates were prepared as described in the previous section. A $300 \mu \mathrm{g}$ aliquot of cell lysate was used for immunoprecipitation using A/G plus agarose beads (Santa Cruz) with either (a) $2 \mu \mathrm{g}$ of the appropriate primary antibody $(\alpha$-ROCK2, $\alpha$-SorL1) or (b) IgG control antibody according to the manufacturers instructions. Equal volumes of IgG and $\alpha$-SorL1/ $\alpha$ ROCK2 immunoprecipitation were separated by SDS PAGE, transferred to PVDF and immunoblotted with $\alpha$-SorL1 (BD Biosciences) and $\alpha$-ROCK2 antibodies as indicated.

\section{Lentivirus production and transduction of N2aAPPSwe $\triangle 9$ PS1 cells}

Lentivirus was produced in the HEK 293 cells as previously described [34], with a few modifications. In brief, the HEK cells were plated onto twelve, $15 \mathrm{~cm}$ petriplates in DMEM supplemented with 10\% FBS, penicillin, and streptomycin. The cells were grown until $60 \%$ confluent and co-transfected with three plasmids. Per petriplate, approximately $57 \mu \mathrm{g}$ of plasmid DNA was used for the transfection: $19 \mu \mathrm{g}$ of the CMV-driven HIV gagpol, tat and rev plasmid [35], $13 \mu \mathrm{g}$ of the CMV-driven vesicular stomatitis virus-G envelope (VSV-G) plasmid (plasmid kindly provided by Dr. Anthony W.S. Chan, Emory). The shRNA expressing lentivectors were prepared by Cellogenetics, Inc. (Gaithersburg, MD) and $25 \mu \mathrm{g}$ of the scramble shRNA (5'-GTTCTCCGAACGTGTCACG-3') lentivector or the ROCK2 shRNA (5'-CAATGAAGCTTCTTAGTAA-3') expression lentivector was used. The media was replaced 24 hours following the transfection with the 3 plasmids and the supernatant was harvested 48 hours later. The supernatant was filtered using a $0.45 \mathrm{~mm}$ pore-sized membrane and concentrated by centrifuging at $10,000 \times \mathrm{g}$ for 2 hours at $4^{\circ} \mathrm{C}$. Lentiviral titres were determined by infecting the HEK 293 cells with varying concentrations of the lentivirus in the presence of Polybrene $(8 \mathrm{mg} / \mathrm{ml}$; Sigma Aldrich). The N2aAPPSwe. $\triangle \mathrm{PS} 1$ cells were infected at an MOI of 10 in the presence of polybrene.

$72 \mathrm{hrs}$ post transduction with sh ROCK2 or scramble lentivirus, cells were collected in ice-cold PBS, centrifuged at $55 \times g$ at $4{ }^{\circ} \mathrm{C}$ for $15 \mathrm{~min}$, and the media were collected and snap frozen lysates prepared as described above. The lysate membrane was analyzed by western blot using pAb369 (APP C-terminal) to detect APP holoprotein (holoAPP) and $\alpha$-actin were detected with the respective relevant primary antibodies, followed by HRP-conjugated, species-specific secondary antibodies. Media gels were immunoblotted with 6E10 to detect sAPP and A $\beta$. Signals were detected using enhanced chemiluminescence (Pierce). Digital images were captured using LAS3000 (FujiFilm), and analyzed using Multi Gauge v3.1 software.

\section{Statistical analysis}

Densitometric analysis of western blot bands ( $D=$ $[B-A U] / \mathrm{mm}^{2}$ ) was performed using Multigauge v3.1 software (Fujifilm). Levels of ROCK were normalized to GFP and expressed as percent of control. Total A $\beta$ levels were analyzed by western blot and bands were normalized to percent control (empty vector). Absolute $A \beta 40$ and $A \beta 42$ concentrations were quantitatively determined by sandwich ELISA (Wako). Independent samples t-tests (parametric design) were utilized to determine significant mean differences between groups. Significance for $\mathrm{t}$-tests are reported with a $\mathrm{p}<0.05$ using two-tailed tests with an $\alpha$-level of 0.05 . All statistical analysis was performed using SPSS v18.0 (SPSS Inc, Chicago, IL).

\section{Abbreviations}

AD: Alzheimer's disease; APP: Amyloid precursor protein; AB: Amyloid beta; TGN: trans Golgi Network; PKC: Protein kinase C; PKA: Protein kinase A; CK2; casein kinase 2; ROCK: rho associated coiled coil kinase; PP1: protein phosphatase 1; PP2A protein phosphatase 2A; sAPPa: soluble amyloid precursor protein alpha; $S A P P B$ : soluble amyloid precursor protein beta; sSorL1: soluble SorL1; CTF: C terminal fragment; BACE: beta amyloid cleavage enzyme; AICD: amyloid intracellular domain; OA: okadaic acid; SDS PAGE: sodium dodecyl sulfate polyacrylamide gel electrophoresis; CIP: calf intestine alkaline phosphatase; Vps10: vacuolar protein sorting 10; GPCRK1: G protein coupled receptor kinase 1; MEF: mouse embryonic fibroblast; N2a: Neuroblastoma; HEK: human embryonic kidney; MOI: multiplicity of infection; ELISA: enzyme linked immunosorbant assay

\section{Acknowledgements}

The authors thank Dr Jim Lah for generously providing $\alpha$-SorL1 $\mathrm{N}$ terminal antibody and wildtype MEF cell lines, and Dr Gopal Thinakaran for providing N2a APPSwe $\triangle 9 P S 1$ cell lines used in this study. 


\section{Author details}

'Department of Neurology, Mount Sinai School of Medicine, New York NY 10029, USA. ${ }^{2}$ Department of Surgery, University of Texas Southwestern, Dallas TX 75390, USA. ${ }^{3}$ Department of Neurology, Taub Center for Research on the Aging Brain, Columbia University College of Physicians and Surgeons, New York NY 10032, USA. ${ }^{4}$ Department of Pediatrics, Mount Sinai School of Medicine, New York NY 10029, USA. ${ }^{5}$ Department of Genetics and Genomic Sciences, Mount Sinai School of Medicine, New York NY 10029, USA. 'Department of Psychiatry, Alzheimer's Disease Research Center, New York NY 10029, USA. 'James J Peters VA Medical Center, Bronx NY 10468, USA.

\section{Authors' contributions}

RL and SG conceived the strategic approach and wrote the paper, MEE and SS additionally contributed to the strategic approach and editing of the manuscript. RL and JWG performed the experiments. All authors have read and approved the manuscript. The work was funded by P01AG10491 to SG and P50AG05138 to Mary Sano, and AG025161 and Alzheimer's association T SAS.

\section{Competing interests}

The authors declare that they have no competing interests.

Received: 18 December 2010 Accepted: 30 December 2010 Published: 30 December 2010

\section{References}

1. Gandy S, Greengard P: Amyloidogenesis in Alzheimer's disease: Some possible therapeutic opportunities. Trends Pharmacol Sci 1992 13:108-113.

2. Small SA, Gandy S: Sorting through the cell biology of Alzheimer's disease: Intracellular pathways to pathogenesis. Neuron 2006, 52:15-31

3. Suzuki T, Nakaya T: Regulation of APP by phosphorylation and protein interactions. J Biol Chem 2008, 283:29633-7.

4. Gandy S, Czernik AJ, Greengard P: Phosphorylation of Alzheimer disease amyloid precursor peptide by protein kinase $\mathrm{C}$ and $\mathrm{Ca2}+/ \mathrm{calmodulin}-$ dependent protein kinase II. Proc Natl Acad Sci USA 1988, 85:6218-6221.

5. Buxbaum JD, Gandy SE, Cicchetti P. Ehrlich ME, Czernik AJ, Fracasso RP, Ramabhadran TV, Unterbeck AJ, Greengard P: Processing of Alzheimer beta/A4 amyloid precursor protein: Modulation by agents that regulate protein phosphorylation. Proc Natl Acad Sci USA 1990, 87:6003-6006.

6. Buxbaum JD, Koo EH, Greengard P: Protein phosphorylation inhibits production of Alzheimer amyloid beta/A4 peptide. Proc Natl Acad Sci USA 1993, 90:9195-9198.

7. Caporaso GL, Gandy SE, Buxbaum JD, Ramabhadran TV, Greengard P: Protein phosphorylation regulates secretion of Alzheimer beta/A4 amyloid precursor protein. Proc Natl Acad Sci USA 1992, 89:3055-3059.

8. Ikin AF, Causevic M, Pedrini S, Benson LS, Buxbaum JD, Suzuki T, Lovestone S, Higashiyama S, Mustelin T, Burgoyne RD, Gandy S: Evidence against roles for phorbol binding protein Munc13-1, ADAM adaptor eve1 , or vesicle trafficking phosphoproteins Munc18 or NSF as phosphostate-sensitive modulators of phorbol/PKC-activated Alzheimer APP ectodomain shedding. Mol Neurodegener 2007, 2:23.

9 Seeger M, Nordstedt C, Petanceska S, Kovacs DM, Gouras GK, Hahne S, Fraser P, Levesque L, Czernik AJ, George-Hyslop PS, Sisodia SS, Thinakaran G, Tanzi RE, et al: Evidence for phosphorylation and oligomeric assembly of presenilin 1. Proc Natl Acad Sci USA 1997, 94:5090-5094

10. Desdouits-Magnen J, Desdouits F, Takeda S, Syu LJ, Saltiel AR, Buxbaum JD, Czernik AJ, Nairn AC, Greengard P: Regulation of secretion of Alzheimer amyloid precursor protein by the mitogen-activated protein kinase cascade. J Neurochem 1998, 70:524-530.

11. Flajolet M, He G, Heiman M, Lin A, Nairn AC, Greengard P: Regulation of Alzheimer's disease amyloid-beta formation by casein kinase I. Proc Nat Acad Sci USA 2007, 104:4159-4164.

12. Miyazawa K, Kitamura N: A scaffold protein JIP-1b enhances amyloid precursor protein phosphorylation by JNK and its association with kinesin light chain 1. J Biol Chem 2003, 268(25):22946-55.

13. Kuo LH, Hu MK, Hsu WM, Tung YT, Wang BJ, Tsai WW, Yen CT, Liao YF: Tumor necrosis factor-alpha-elicited stimulation of $y$-secretase is mediated by c-jun $\mathrm{N}$-terminal kinase-dependent phosphorylation of presenilin and nicastrin. Mol Biol Cell 2008, 19:4201-4212.
14. Pedrini S, Carter TL, Prendergast G, Petanceska S, Ehrlich ME, Gandy S: Modulation of statin-activated shedding of Alzheimer APP ectodomain by ROCK. PLoS Med 2005, 2:e18.

15. Vieira SI, Rebelo S, Esselmann H, Wiltfang J, Lah J, Lane R, Small SA, Gandy S, da Cruz E Silva EF, da Cruz E Silva OA: Retrieval of the Alzheimer's amyloid precursor protein from the endosome to the TGN is S655 phosphorylation state-dependent and retromer-mediated. Mol Neurodegener 2010, 5:40.

16. Le Gall SM, Maretzky T, Issuree PD, Niu XD, Reiss K, Saftig P, Khokha R, Lundell D, Blobel CP: ADAM17 is regulated by a rapid and reversible mechanism that controls access to its catalytic site. J Cell Sci 2010, 123:3913-22.

17. Walter J, Fluhrer R, Hartung B, Willem M, Kaether C, Capell A, Lammich S, Multhaup G, Haass C: Phosphorylation regulates intracellular trafficking of beta-secretase. J Biol Chem 2001, 276:14634-41.

18. Rebelo S, Vieira SI, Esselmann H, Wiltfang J, da Cruz e Silva EF, da Cruz e Silva OA: Tyr687 dependent APP endocytosis and abeta production. J Mol Neurosci 2007, 32:1-8.

19. Tamayev R, Zhou D, D'Adamio $L$ : The interactome of the amyloid beta precursor protein family members is shaped by phosphorylation of their intracellular domains. Mol Neurodegener 2009, 4:28.

20. Small SA, Kent K, Pierce A, Leung C, Kang MS, Okada H, Honig L, Vonsattel JP, Kim TW: Model-guided microarray implicates the retromer complex in Alzheimer's disease. Ann Neurol 2005, 58:909-19.

21. Andersen O, Fjorback A, Seaman M, Ilsoe E, Gustafsen C, Willnow T, Madsen P, Nykjaer A: The role of the retromer in SorLA dependent APP transport and processing. Alzheimer's and Dementia 2010, 6:S101.

22. Lane RF, Raines SM, Steele JW, Ehrlich ME, Lah JA, Small SA, Tanzi RE, Attie AD, Gandy S: Diabetes-associated SorCS1 regulates Alzheimer's amyloid-beta metabolism: Evidence for involvement of SorL1 and the retromer complex. J Neurosci 2010, 30:13110-13115.

23. Nyborg AC, Ladd TB, Zwizinski CW, Lah JJ, Golde TE: Sortilin, SorCS1b, and SorLA Vps10p sorting receptors, are novel gamma-secretase substrates. Mol Neurodegener 2006, 1:3

24. Andersen OM, Reiche J, Schmidt V, Gotthardt M, Spoelgen R, Behlke J, von Arnim CA, Breiderhoff T, Jansen P, Wu X, Bales KR, Cappai R, Masters CL, et al: Neuronal sorting protein-related receptor sorLA/LR11 regulates processing of the amyloid precursor protein. Proc Natl Acad Sci USA 2005, 102:13461-13466

25. Offe K, Dodson SE, Shoemaker JT, Fritz JJ, Gearing M, Levey Al, Lah JJ: The lipoprotein receptor LR11 regulates amyloid beta production and amyloid precursor protein traffic in endosomal compartments. J Neurosci 2006, 26:1596-1603.

26. Nielsen MS, Gustafsen C, Madsen P, Nyengaard JR, Hermey G, Bakke O, Mari M, Schu P, Pohlmann R, Dennes A, Petersen CM: Sorting by the cytoplasmic domain of the amyloid precursor protein binding receptor SorLA. Mol Cell Biol 2007, 27:6842-6851.

27. Schmidt V, Sporbert A, Rohe M, Reimer T, Rehm A, Andersen OM, Willnow TE: SorLA/LR11 regulates processing of amyloid precursor protein via interaction with adaptors GGA and PACS-1. J Biol Chem 2007, 282:32956-32964

28. Amanchy R, Periaswamy B, Mathivanan S, Reddy R, Tattikota SG, Pandey A A curated compendium of phosphorylation motifs. Nat Biotechnol 2007, 25:285-286.

29. Nakagawa O, Fujisawa K, Ishizaki T, Saito Y, Nakao K, Narumiya S: ROCK-I and ROCK-II, two isoforms of rho-associated coiled-coil forming protein serine/threonine kinase in mice. FEBS Lett 1996, 392:189-193.

30. Herskowitz JH, Seyfried NT, Gearing M, Kahn RA, Peng J, Levey Al, Lah JJ: RHO kinase II phosphorylation of the lipoprotein receptor LR11/SorLA alters amyloid-beta production. J Biol Chem 2010, (PMID:21147781).

31. Zhou Y, Su Y, Li B, Liu F, Ryder JW, Wu X, Gonzalez-DeWhitt PA, Gelfanova V, Hale JE, May PC, Paul SM, Ni B: Nonsteroidal antiinflammatory drugs can lower amyloidogenic Abeta42 by inhibiting rho. Science 2003, 302:1215-1217.

32. Leuchtenberger $S$, Kummer MP, Kukar T, Czirr E, Teusch N, Sagi SA, Berdeaux R, Pietrzik CU, Ladd TB, Golde TE, Koo EH, Weggen S: Inhibitors of rho-kinase modulate amyloid-beta (abeta) secretion but lack selectivity for Abeta42. J Neurochem 2006, 96:355-365.

33. Muhammad A, Flores I, Zhang H, Yu R, Staniszewski A, Planel E, Herman M, Ho L, Kreber R, Honig LS, Ganetzky B, Duff K, Arancio O, Small SA: Retromer deficiency observed in Alzheimer's disease causes hippocampal 
dysfunction, neurodegeneration, and Abeta accumulation. Proc Natl Acad Sci USA 2008, 105:7327-32.

34. Naldini L, Blomer U, Gage FH, Trono D, Verma IM: Efficient transfer, integration, and sustained long-term expression of the transgene in adult rat brains injected with a lentiviral vector. Proc Natl Acad Sci USA 1996, 93:11382-11388.

35. Dull T, Zufferey R, Kelly M, Mandel RJ, Nguyen M, Trono D, Naldini L: A third-generation lentivirus vector with a conditional packaging system. J Virol 1998, 72:8463-8471.

doi:10.1186/1750-1326-5-62

Cite this article as: Lane et al:: Protein kinase $C$ and rho activated coiled coil protein kinase 2 (ROCK2) modulate Alzheimer's APP metabolism and phosphorylation of the Vps10-domain protein, SorL1. Molecular

Neurodegeneration 2010 5:62

Submit your next manuscript to BioMed Central and take full advantage of:

- Convenient online submission

- Thorough peer review

- No space constraints or color figure charges

- Immediate publication on acceptance

- Inclusion in PubMed, CAS, Scopus and Google Scholar

- Research which is freely available for redistribution

Submit your manuscript at www.biomedcentral.com/submit
C Biomed Central 